in vivo $34: 1875-1882(2020)$

doi:10.21873/invivo.11983

\title{
Radiological and Histopathological Features of Internal Tooth Resorption
}

\author{
TILL KOEHNE ${ }^{1}$, JOZEF ZUSTIN ${ }^{2}$, MICHAEL AMLING ${ }^{2}$ and REINHARD E. FRIEDRICH ${ }^{3}$ \\ ${ }^{1}$ Department of Orthodontics, University Medical Center Hamburg-Eppendorf, Hamburg, Germany; \\ ${ }^{2}$ Department of Osteology and Biomechanics, University Medical Center Hamburg-Eppendorf, Hamburg, Germany; \\ ${ }^{3}$ Department of Oral and Maxillofacial Surgery, \\ University Medical Center Hamburg-Eppendorf, Hamburg, Germany
}

\begin{abstract}
Background: Internal root resorption is an endodontic disease characterized by progressive resorption of dentin from the inside of the pulp chamber. It is a comparatively rare finding in the permanent dentition, and the underlying pathology is not fully understood. Case Report: A 45-year-old patient was referred to our Department for the evaluation of the lower right canine and the upper left wisdom tooth. Pulp sensitivity tests, cone-beam tomography, and magnetic resonance imaging were used to determine the extent of lesions of the affected teeth. The teeth were subsequently extracted due the extent of the lesions. The same was the case for the upper right canine, which developed a severe internal resorption 10 months later. Micro-computed tomography of the extracted teeth revealed that all lesions had a well-defined border with no evidence of sclerosis or hypomineralization. Pulp stones were evident inside the pulp chamber. Ground sectioning of the upper right canine revealed pulp necrosis and an acute infection that had gradually moved in the apical direction. Large multi-nucleated resorbing cells were found on the dentin surface. Importantly, the apical half of the pulp exhibited comparatively normal tissue without substantial inflammatory changes. Decalcified histology of the upper left wisdom tooth demonstrated a completely different histopathological appearance characterized by chronically inflamed granulation tissue with pseudoepitheliomatous
\end{abstract}

This article is freely accessible online.

Correspondence to: Dr. Till Koehne, DMD, Ph.D., Department of Orthodontics, University Medical Center Hamburg-Eppendorf, Martinistr. 52, 20246 Hamburg, Germany. Tel: +49 40741054398, Fax: +49 40741054887, e-mail: tkoehne@uke.de

Key Words: Magnetic resonance imaging, cone beam computed tomography, micro-computed tomography, pulp histology, pulp inflammation, internal root resorption. hyperplasia and massive bacterial colonization. Conclusion: Our analyses demonstrate that internal root resorption is a multifaceted dental disease with considerable variability in the rate of the underlying inflammatory changes. Oral surgeons should take this into consideration when evaluating the need for extraction of teeth with internal root resorption.

Resorption of calcified tissues, such as bone, dentin, and cementum, is a prerequisite for skeletal homeostasis and tooth eruption. Resorption of permanent teeth, however, is a pathological condition that may result in the loss of the affected teeth. Tooth resorption can occur externally on the root surface or internally within the pulp chamber. External and internal tooth resorption should be considered as discrete entities which differ in terms of prevalence and etiology.

External tooth resorption may affect up to $5 \%$ of all permanent teeth, and it is usually caused by trauma, ectopic teeth, or orthodontic tooth movement (1). In contrast, internal tooth resorption (IR) is a comparatively rare finding, and its etiology has not been fully elucidated (2). IR is predominantly a lesion within the upper third of the pulp chamber and may affect both the crown and the root of the tooth. The initial finding is resorption of the inner wall of the root canal that progresses in a centrifugal direction. Accompanying changes such as the resorption of the root surface or the alveolar bone may occur, but are not mandatory findings in IR. In fact, the diagnosis of IR can only be made if external factors such as root caries or cervical resorptions can be excluded.

There are several studies describing radiological and histological features of teeth with IR (3-7). However, some of these reports need to be interpreted with caution, since they were based on two-dimensional radiographs, where internal and external resorption can often not be clearly distinguished. Moreover, only a few reports performed a thorough histological analysis.

The aim of this study of a case was therefore to describe three-dimensional radiographic characteristics of teeth with 

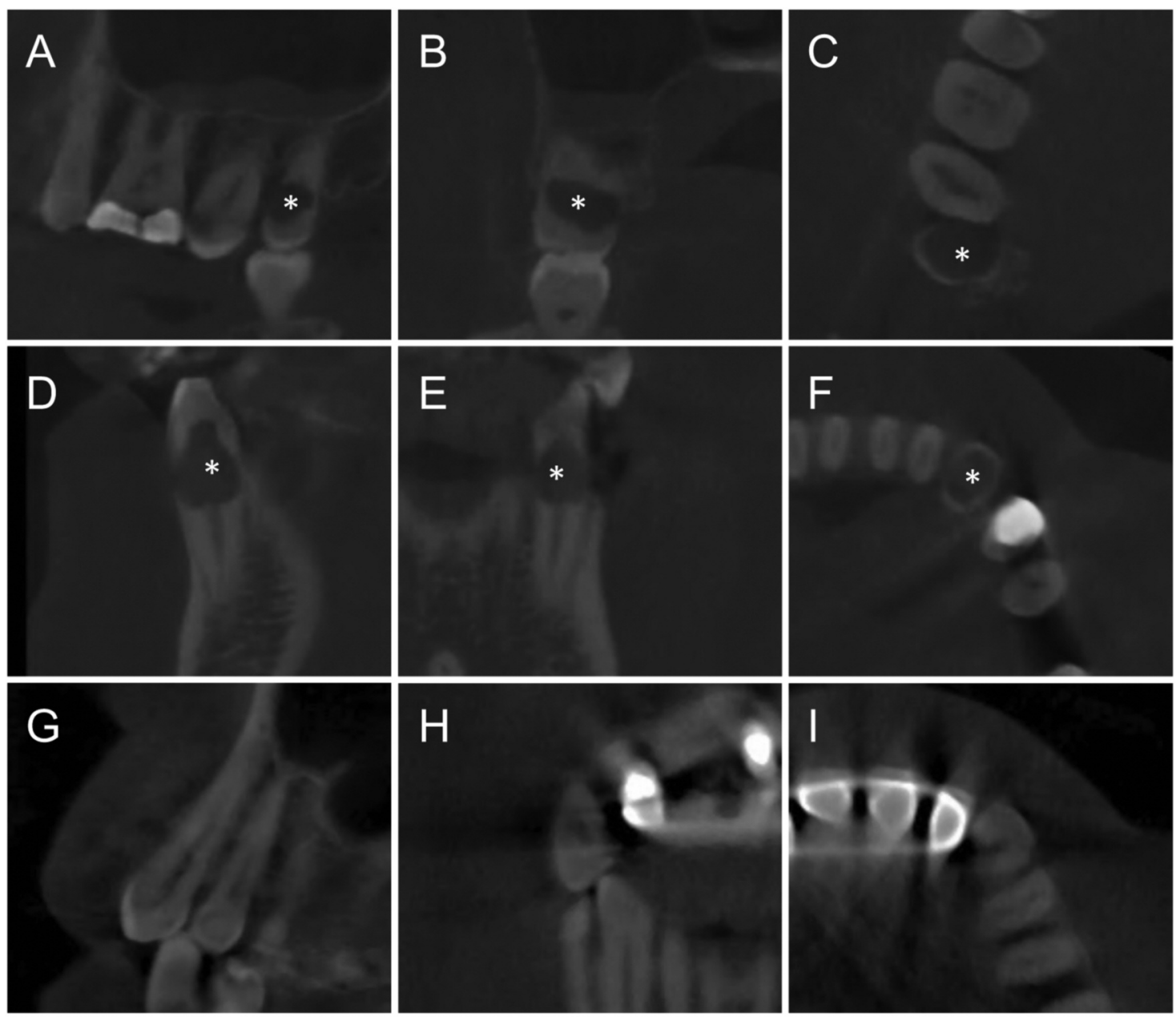

Figure 1. Cone-beam computed tomography. Reconstructed views (sagittal, axial, and coronal) demonstrating large, well-defined radiolucencies within the pulp chambers of the upper left wisdom tooth $(A-C)$ and the lower right canine $(D-F)$. Note that at this time point, there was only a small lateral lesion of the upper right canine (G-I), which might also be an artifact.

IR using cone-beam tomography (CBCT), magnetic resonance imaging (MRI), and micro-computed tomography (micro-CT). In addition, we performed decalcified histology and ground sectioning to describe the different histopathological stages of IR.

\section{Case Report}

A 45-year-old man was referred to the Department of Oral and Maxillofacial Surgery, University Medical Center Hamburg-Eppendorf, for evaluation of resorption of the lower right canine and the upper left wisdom tooth. The medical history was non-contributory. A review of the dental history revealed that the patient had been involved in an assault about 15 years earlier that resulted in a fracture of the upper left central incisor. The tooth had been restored by a general dentist.
The oral examination revealed that the lower first and second molars, as well as the lower left first bicuspid, were missing. Pulp sensitivity tests (Henry Schein, Langen, Germany) produced no response from the lower right canine and the upper left wisdom tooth, whilst all other teeth responded to cold without lingering. No caries were clinically detected.

CBCT was performed using a CBCT scanner (3D Accuitomo 170, MCT-1 EX-1 F17; Morita MFG Corp., Kyoto, Japan). Imaging was carried out with an exposure time of 18 seconds $\left(360^{\circ}\right)$, slice thickness of $1 \mathrm{~mm}$, voxel size of $80-250 \mu \mathrm{m}$, and exposure volume of $170 \times 120 \mathrm{~mm}$. $\mathrm{CBCT}$ of the jaws indicated resorption within the lower right canine and upper left wisdom teeth that were connected to the surrounding periodontal ligament (Figure 1A-F). The upper right canine, which was also later affected by IR, was unremarkable at this stage (Figure 1G-I). 

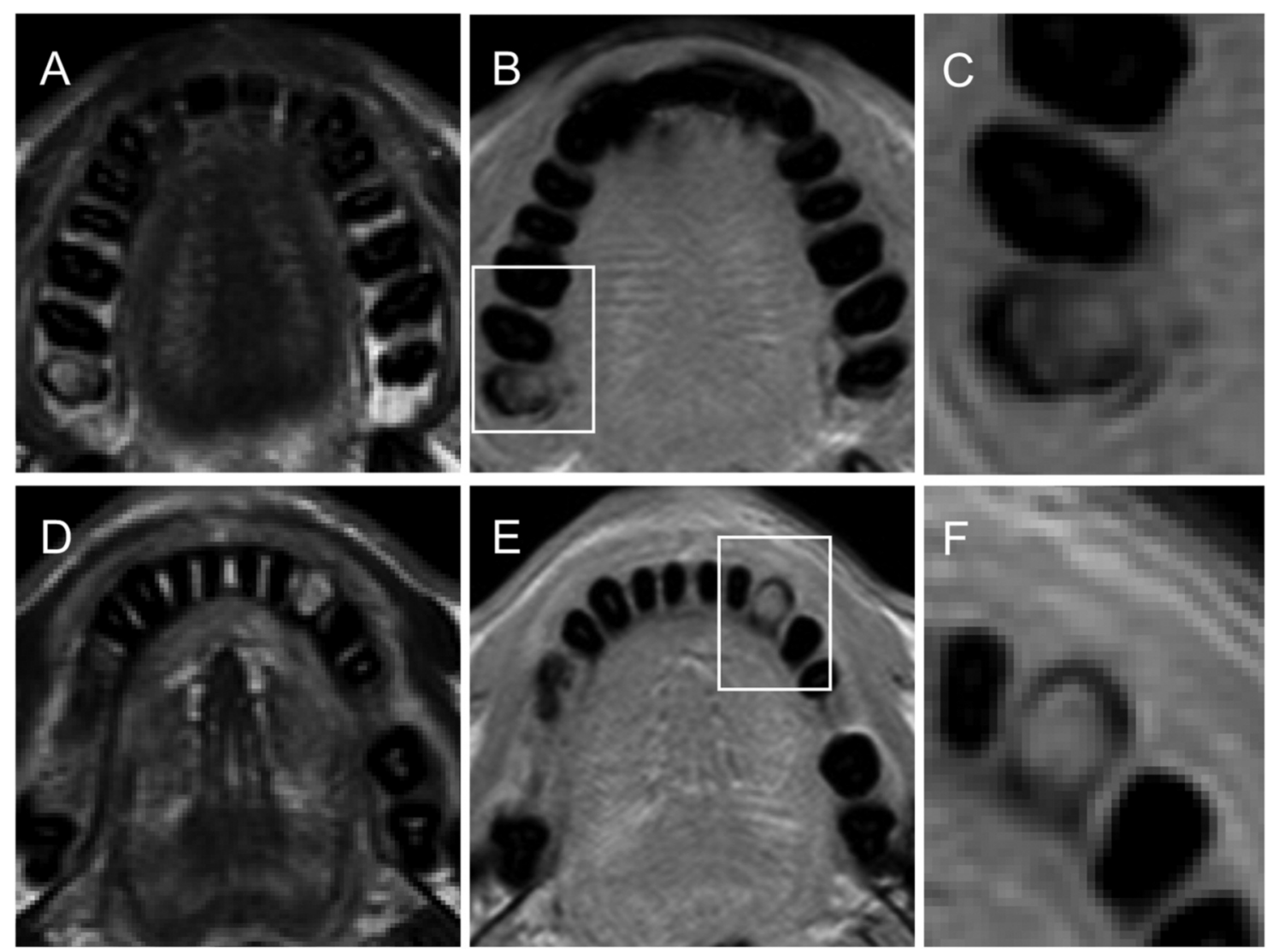

Figure 2. Magnetic resonance imaging. Axial T2-weighted images of the upper $(A-C)$ and lower $(D-F)$ jaws revealed abnormal high signal intensity inside the upper left wisdom tooth and the lower right canine. The regions marked by the white rectangles in $B$ and $E$ are shown at higher magnifications in $C$ and $F$, respectively.

MRI has proven to be a sensitive method for detecting changes in pulp vascularization (8). It was therefore used to examine the vascularization of the diseased teeth and to exclude a general disorder of pulp vascularization (Figure 2). The healthy, vital teeth produced a slightly hyperintense signal of threadlike (coronal or sagittal section) or point-like (axial section) shape, which was homogeneously surrounded by a non-stimulated (black) region identified as the dental hard tissues. MRI signals of the pulp at the site of the IR differed from the control teeth both in shape and intensity. In the axial cross-section, the hyperintense signal was present in almost the entire crown area. The signal was significantly more intense in the area of the inflammatory pulp as compared to those of the vital teeth. Irregularly distributed hypointense signals were present within these hyperintense regions. The IR lesions were also hyperintense in short-tau inversion recovery, and T1- and T2-weighted sequences, and gave positive signals in diffusion-weighted whole-body imaging with background body signal suppression (data not shown).

Due to the extent of the lesions and the perforation, extraction of the lower right canine and the upper left wisdom tooth were indicated. Patient consent was obtained after a comprehensive discussion of the complications and risks of surgical treatment and the need for prosthetic rehabilitation.

The extraction of both teeth was performed under local anesthesia without complication, although the teeth fractured. A partial ankylosis of the lower right canine was noted. A part of the pulp and the surrounding periodontal tissue were preserved for histological analysis. The healing of the wounds was uneventful. Ten months later, the patient returned to the Outpatient Department for evaluation of large IR within the upper right canine. The tooth was considered as not worth preserving after the evaluation of a radiograph performed by the patient's general dentist (not shown). The extraction of the tooth was performed under local anesthesia without complication. It was possible to extract the tooth in one piece. A lateral perforation at the cervix was noted (Figure 3A).

The extracted teeth were fixed in $3.7 \%$ phosphate-buffered saline-buffered formaldehyde. Contact radiography (Faxitron ${ }^{\circledR}$ MX-20, Faxitron Bioptics, Hennef, Germany) of 

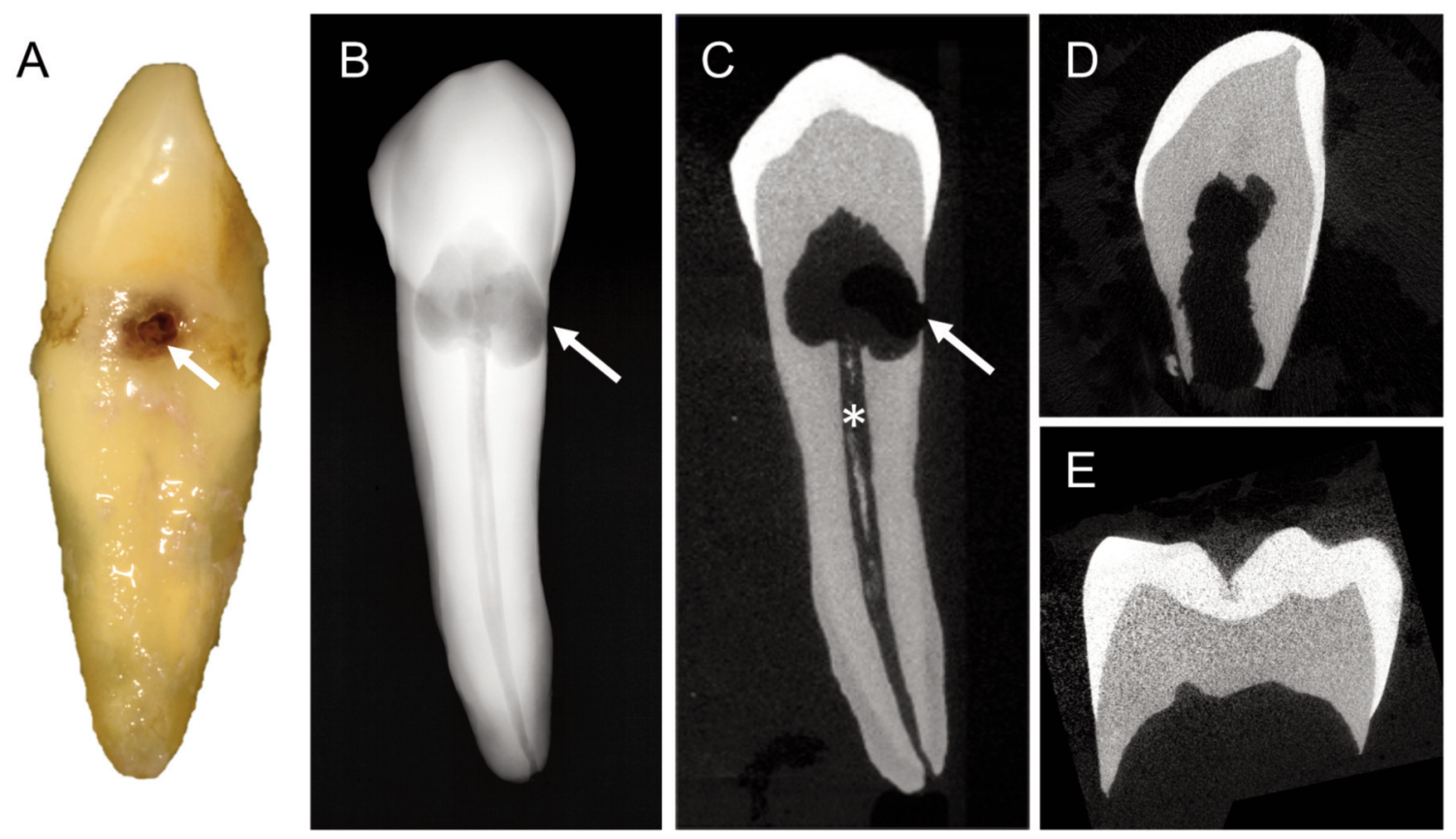

Figure 3. Ex-vivo specimens imaging. Photography $(A)$ and contact radiography $(B)$ of the upper right canine. A small perforation is evident at the tooth cervix (arrow). Micro-computed tomography of all extracted teeth $(C-E)$. Note the fine lines of calcification within the pulp canal of the upper right canine (asterisk).

the upper right canine revealed a large centric radiolucent lesion with well-defined borders (Figure 3B). Micro-CT ( $\mu$ CT-40; Scanco Medical AG, Wangen-Brüttisellen, Switzerland) was further performed to analyze the threedimensional morphology of the resorption areas (Figure 3CE). All lesions had a well-defined linear or scalloped border. There was no evidence of sclerosis or demineralization of the neighboring dentin. However, a small line of calcification was noted within the root canal of the upper right canine (Figure 3C).

Ground sectioning of the upper right canine was performed for histological characterization of the internal root resorption. The tooth was embedded undecalcified in methyl methacrylate-based resin (Technovit 7200 VLZ; Kulzer, Wehrheim, Germany), ground sectioned, and stained with toluidine blue as described previously (9). Different degrees of inflammation were found in the upper half of the pulp (Figure 4A-C). The regions in close proximity to the lesion were characterized by necrotic tissue with purulent exudate and bacterial colonization (Figure 4D). The necrotic area was surrounded by granulocyte-rich granulation tissue with focal areas of dense neutrophilic infiltration and micro-abscess formation (Figure 4E and F). Resorption lacunae with multi-nucleated odontoclasts (arrow in Figure 4F) were found on the dentin surface. The neighboring apical pulp showed histopathological signs of acute inflammation characterized by the presence of plasma cells, histiocytes, and lymphocytes (Figure 4G). The apical half of the pulp exhibited normal tissue without substantial inflammatory changes or necrosis (Figure $4 \mathrm{H}$ ). Numerous calcified pulp stones within the root canal (Figure 4I) were remarkable. These were found both in areas with high degrees of inflammation as well as in areas without histopathological signs of inflammation. Taken together, the histological appearance of this tooth with IR was characterized by pulp necrosis and an acute infection that gradually moved in the apical direction.

The upper left wisdom tooth was embedded decalcified in order to further analyze the histological appearance of internal root resorption. The tissue was decalcified (USEDECALC, Medite GmbH, Burgdorf, Germany), waxembedded, serially sectioned, and stained with hematoxylin and eosin. The specimen consisted of pulpal tissue, dentin, periodontal ligament, and alveolar bone (Figure 5A). The pulp was filled with chronically inflamed granulation tissue (Figure 5B) and inflammatory-altered stratified squamous epithelium with scattered apoptotic cells (Figure 5C). Squamous epithelium also lined the pulpal side of the dentin layer (Figure 5D). The scalloped shape of the dentin indicated previous focal resorption. Taken together, the histological appearance of this tooth was characterized by granulation tissue with pseudoepitheliomatous hyperplasia and bacterial colonization. 

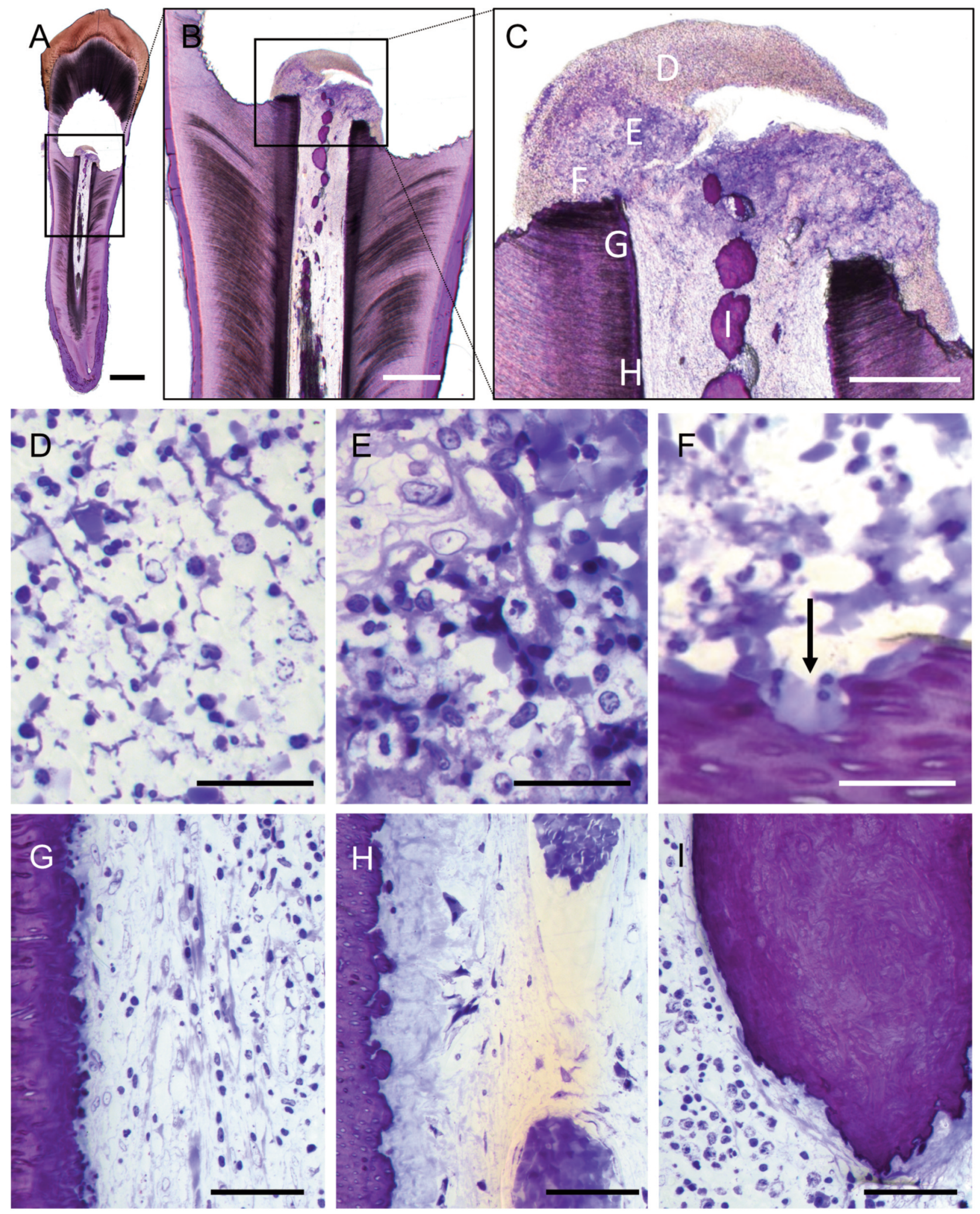

Figure 4. Microscopy of a toluidine blue-stained ground section through an undecalcified prepared tooth. Full overview (A) of the upper right canine and high-power images $(B, C)$ of the regions marked by the black rectangles. The remaining pulp exhibits different stages of inflammation and necrosis, which are shown in images at higher magnifications (D-I). Scale bar: A: $2.5 \mathrm{~mm} ; \mathrm{B}: 1 \mathrm{~mm} ; \mathrm{C}: 0.5 \mathrm{~mm} ; \mathrm{D}-\mathrm{F}: 20$ um and G-I: 50 um. 

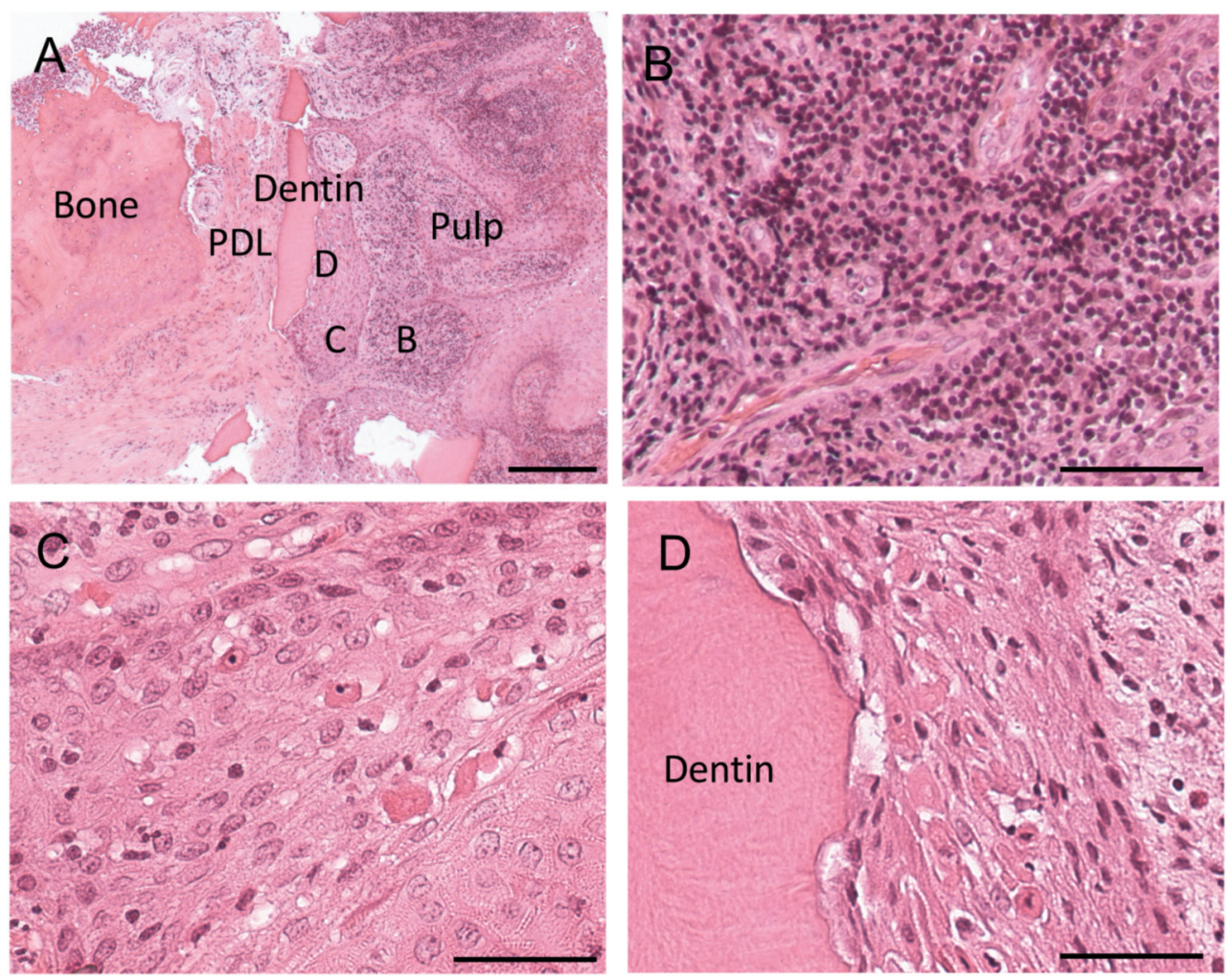

Figure 5. Microscopy of hematoxylin-eosin-stained sections of pulp, periodontal ligament tissue (PDL), and adjacent gingiva from the upper left wisdom tooth $(A-D)$. Regions noted in panel A are shown at a higher magnification in B-D. Scale bar: A: $250 \mu m ; B-D: 50 \mu m$.

\section{Discussion}

This report presents a comprehensive radiological and histological analysis of teeth with IR. From a clinical perspective, we demonstrate that MRI can be a helpful tool in addition to CBCT for the clinical imaging of IR. From a basic science perspective, we show that the histological appearance of IR of a tooth can differ remarkably from one specimen to another.

Previous studies reported various histological findings of human teeth with IR (3-6). One reason for this heterogeneity may be that IR is a localized process that only affects a part of the pulp. It was therefore important to use ground sectioning to analyze a tooth with IR along its entire axis. We observed that the severity of inflammation decreased along a coronal-to-apical gradient. The coronal center of the lesion was characterized by necrosis with bacterial colonization. However, it is likely that most of the necrotic tissue was liquefactive and lost during the fixation process. The surrounding areas showed signs of acute inflammation and were mainly composed of granulation tissue with microabscesses and dense neutrophilic infiltration. The tissue farther away from the lesions still contained inflammatory cells, such as plasma cells, histiocytes, and lymphocytes.
However, the apical half of the pulp exhibited relatively normal organized soft tissue. These observations are in line with previous studies and demonstrate that the histological appearance of the pulp with IR is similar to that of irreversible pulp inflammation $(3,5)$.

The histological appearance of the tooth shown in Figure 5 with root IR was remarkably different from that of the tooth shown in Figure 4. It was mainly characterized by chronically inflamed granulation tissue. We did not observe periodontal connective tissue inside the pulp, although there were several perforations of the periodontal ligament. Therefore, our data do not support the assumption that the presence of membranelike periodontal connective tissue is a prerequisite for internal root resorption (4). An interesting finding, however, was that the granulation tissue was covered by inflammatory-altered squamous epithelium. This epithelial lining resembled pseudoepitheliomatous hyperplasia that has been observed in other oral pathologies such as bisphosphonate-related osteonecrosis of the jaw (10). The source of these epithelial cells is unclear but it is possible that they originate from the gingival epithelium or from epithelial remnants within the periodontal ligament (i.e. rests of Malassez) (11). The histological appearance of this tooth with IR is somewhat similar to that of teeth with hyperplastic pulpitis (also called 
irreversible chronic open pulpitis) (12). Taken together, our findings indicate that the histological appearance of teeth with IR is characterized by irreversible chronic pulpitis with necrosis and pseudoepitheliomatous hyperplasia.

The temporospatial changes in the inflammatory process are important for the interpretation of the presented findings. In fact, it is remarkable that we have clinical and radiological records of one tooth that was unsuspicious at the first consultation and exhibited large IR only 10 months later. This indicates that IR can be a rapidly progressing disease.

A unique histological feature of IR is the presence of dentinoclastic cells within the root channel (2). We also observed these large multi-nucleated cells on the dentin surface of the tooth with IR (Figure 4F). However, these cells were not found in the tooth with IR shown in Figure 5. In contrast, numerous bacteria were present on the dentin surface. It is therefore important to mention that bacteria can also directly resorb mineralized matrix as seen in osteomyelitis (13). In fact, the reason for massive bacterial colonization is unclear in our case. There was no evidence of caries in the clinical and radiographic examination. The microradiography and microCT also showed no signs of caries or hypomineralization. Importantly, the micro-CT also revealed no evidence of microcracks in the enamel or dentin layer. Dental traumas causing micro-cracks in the tooth structure are regarded as one cause of IR (13). Our patient was indeed involved in an assault but this event had happened about 15 years before any IR was detected. Moreover, the localization of IR in the canines and the maxillary third molar are not indicative of a traumatic pathogenesis. Taken together, it seems unlikely that trauma was the main cause of IR in our case.

Another unusual finding in our case is that multiple teeth were affected, both simultaneously and consecutively. In fact, there are only a few reports in the literature of cases with multiple IR (14-16). The occurrence of IR in monozygotic twins further suggests that genetic disposition may play a role in its pathogenesis (17).

Regardless of the pathogenesis, pulpal vitality is a prerequisite for IR. It was therefore of interest to image the teeth with MRI. We observed a strong signal intensity on MRI of the affected teeth. However, this signal should not be interpreted as physiological revascularization of the pulp tissue as observed in MRI images of traumatized teeth (8). In contrast, a strong signal intensity of the pulpal tissue in teeth with IR should be interpreted as a sign of high activity of the pathological process. Notably, the upper right canine, which was later affected by IR, was inconspicuous on MRI at the patient's first consultation at our Department. It therefore remains to be determined whether MRI has any advantage in comparison to CBCT scanning for the diagnosis of root IR despite the fact that it does not require ionizing radiation. In fact, CBCT scanning should be regarded nowadays as standard of care for the radiographic evaluation of IR (2), since internal and cervical root resorption can often not clearly be distinguished on two-dimensional radiographs. The CBCT scan also helps to estimate the extent of the lesion and to detect perforations of the neighboring periodontal ligament. In fact, the large extent of the lesions, as well as the perforations, were in this case the reasons for extracting the teeth and not performing endodontic treatment.

\section{Conclusion}

Our report demonstrates that internal root resorption is a multifaceted endodontic disease with considerable variability in the rate of the inflammatory changes. Clinicians should be aware of this when evaluating treatment options for teeth with IR.

\section{Conflicts of Interest}

Nothing to declare. This research did not receive any specific grant from funding agencies in the public, commercial, or not-for-profit sectors.

\section{Authors' Contributions}

TK and JZ performed the histological and microstructural studies of the specimens; MA performed the microstructural analysis of the specimens. REF treated the patient and initiated the study. All Authors collected and analyzed the data, wrote the article, and gave final approval to publication.

\section{References}

1 Weltman B, Vig KW, Fields HW, Shanker S and Kaizar EE: Root resorption associated with orthodontic tooth movement: A systematic review. Am J Orthod Dentofacial Orthop 137(4): 462476; discussion 412A, 2010. PMID: 20362905. DOI: 10.1016/ j.ajodo.2009.06.021

2 Patel S, Ricucci D, Durak C and Tay F: Internal root resorption: A review. J Endod 36(7): 1107-1121, 2010. PMID: 20630282. DOI: $10.1016 /$ j.joen.2010.03.014

3 Gassmann G and Arnold WH: Case report of an internal granuloma investigated by light and scanning electron microscopy. Head Face Med 11: 20, 2015. PMID: 26065880. DOI: $10.1186 / \mathrm{s} 13005-015-0077-6$

4 Wedenberg $\mathrm{C}$ and Zetterqvist L: Internal resorption in human teeth--a histological, scanning electron microscopic, and enzyme histochemical study. J Endod 13(6): 255-259, 1987. PMID: 3474343. DOI: $10.1016 /$ S0099-2399(87)80041-9

5 Tronstad L: Root resorption--etiology, terminology and clinical manifestations. Endod Dent Traumatol 4(6): 241-252, 1988. PMID: 3078294. DOI: 10.1111/j.1600-9657.1988.tb00642.x.

6 Allen AL and Gutmann JL: Internal root resorption after vital root resection. J Endod 3(11): 438-440, 1977. PMID: 275443. DOI: $10.1016 /$ S0099-2399(77)80119-2

7 Barker BC and Lockett BC: Histology of external and internal resorption. Aust Dent J 22(5): 360-370, 1977. PMID: 277154. DOI: $10.1111 / \mathrm{j} .1834-7819.1977 . t b 05122 . x$ 
8 Assaf AT, Zrnc TA, Remus CC, Khokale A, Habermann CR, Schulze D, Fiehler J, Heiland M, Sedlacik J and Friedrich RE: Early detection of pulp necrosis and dental vitality after traumatic dental injuries in children and adolescents by 3-Tesla magnetic resonance imaging. J Craniomaxillofac Surg 43(7): 1088-1093, 2015. PMID: 26165761. DOI: 10.1016/j.jcms. 2015.06.010

9 Hahn M, Vogel M and Delling G: Undecalcified preparation of bone tissue: Report of technical experience and development of new methods. Virchows Arch A Pathol Anat Histopathol 418(1): 1-7, 1991. PMID: 1899163. DOI: 10.1007/bf01600238

10 Zustin J, Reske D, Zrnc TA, Heiland M, Scheuer HA, Assaf AT and Friedrich RE: Pseudoepitheliomatous hyperplasia associated with bisphosphonate-related osteonecrosis of the jaw. In Vivo 28(1): 125-131, 2014. PMID: 24425847.

11 Ten Cate AR: The epithelial cell rests of malassez and the genesis of the dental cyst. Oral Surg Oral Med Oral Pathol 34(6): 956-964, 1972. PMID: 4509005. DOI: 10.1016/00304220(72)90233-2

12 Caliskan MK, Oztop F and Caliskan G: Histological evaluation of teeth with hyperplastic pulpitis caused by trauma or caries: Case reports. Int Endod J 36(1): 64-70, 2003. PMID: 12656516. DOI: $10.1046 /$ j.1365-2591.2003.00590.x
13 Marriott I: Apoptosis-associated uncoupling of bone formation and resorption in osteomyelitis. Front Cell Infect Microbiol 3: 101, 2013. PMID: 24392356. DOI: 10.3389/fcimb.2013.00101

14 Celikten B, Uzuntas CF and Kurt H: Multiple idiopathic external and internal resorption: Case report with cone-beam computed tomography findings. Imaging Sci Dent 44(4): 315-320, 2014. PMID: 25473640. DOI: 10.5624/isd.2014.44.4.315

15 Lynch EJ and Ahlberg KF: Bilateral idiopathic root resorption of upper first premolars. Int Endod J 17(4): 218-220, 1984. PMID: 6593307. DOI: 10.1111/j.1365-2591.1984.tb00408.x

16 Nagaraj E, Kaur RP, Raghuram PH and Kumar PS: Multiple internal resorption in permanent teeth associated with hyperparathyroidism. Indian J Dent Res 24(1): 128-131, 2013. PMID: 23852246. DOI: 10.4103/0970-9290.114917

17 Mincik J, Urban D and Timkova S: Clinical management of two root resorption cases in endodontic practice. Case Rep Dent 2016: 9075363, 2016. PMID: 27648314. DOI: 10.1155/ $2016 / 9075363$

Received March 2, 2020

Revised March 24, 2020

Accepted March 28, 2020 